Helsinki University of Technology Institute of Mathematics Research Reports

\title{
COMPUTATIONS WITH FINITE ELEMENT METHODS FOR THE BRINKMAN PROBLEM
}

Antti Hannukainen Mika Juntunen Rolf Stenberg 

Helsinki University of Technology Institute of Mathematics Research Reports

\section{COMPUTATIONS WITH FINITE ELEMENT METHODS FOR THE BRINKMAN PROBLEM}

Antti Hannukainen Mika Juntunen Rolf Stenberg 
Antti Hannukainen, Mika Juntunen, Rolf Stenberg: Computations with finite element methods for the Brinkman problem; Helsinki University of Technology Institute of Mathematics Research Reports A569 (2009).

Abstract: Various finite element families for the Brinkman flow (or StokesDarcy flow) are tested numerically. Particularly the effect of small permeability is studied. The tested finite elements are the MINI element, the TaylorHood element, and the stabilized equal order methods. The numerical tests include both a priori analysis and adaptive methods.

AMS subject classifications: $65 \mathrm{~N} 30$

Keywords: Brinkman equation, Stokes equation, Darcy equation, MINI, TaylorHood, stabilized methods, a posteriori, adaptive

\section{Correspondence}

Helsinki University of Technology

Department of Mathematics and Systems Analysis

P.O. Box 1100

FI-02015 TKK

Finland

antti.hannukainen@tkk.fi,mika.juntunen@tkk.fi,rolf.stenberg@tkk.fi

ISBN 978-951-22-9860-0 (print)

ISBN 978-951-22-9861-7 (PDF)

ISSN 0784-3143 (print)

ISSN 1797-5867 (PDF)

Helsinki University of Technology

Faculty of Information and Natural Sciences

Department of Mathematics and Systems Analysis

P.O. Box 1100, FI-02015 TKK, Finland

email: math@tkk.fi http://math.tkk.fi/ 


\section{Introduction}

The Brinkman equations are used in modeling porous media flow in the case of high porosity when shear effects of the fluid has to be taken into account, se e.g. $[20,18,1,2,3]$.

In a recent paper [15] we have studied the finite element approximation of the model. We have proved both a priori and a posteriori estimates for some classes of methods that (in view of the analysis) are robust. The purpose of this paper is to complement the previous paper with numerical benchmark computations.

The plan of the paper is as follows. In the next section we recall the Brinkman equations, write them in a scaled form which shows the mathematical structure of the problem. Section 3 is devoted to the finite element approximation. We recall the results of [15] and the methods presented therein. We also give the corresponding results for the so-called Taylor-Hood family. The main part of the paper is Section 4 in which we give the results of extensive benchmark computations.

\section{The Brinkman problem}

The model consists of the following elliptic system of differential equations

$$
\begin{aligned}
-2 \mu \boldsymbol{A} \boldsymbol{u}+\frac{\mu}{K} \boldsymbol{u}+\nabla p & =\boldsymbol{f} \text { in } \Omega, \\
\operatorname{div} \boldsymbol{u} & =g \text { in } \Omega,
\end{aligned}
$$

where $\boldsymbol{u}$ and $p$ are the velocity and pressure, respectively. Here, we have denoted

$$
\boldsymbol{A} \boldsymbol{u}:=\operatorname{div} \boldsymbol{\varepsilon}(\boldsymbol{u}) \quad \text { with } \boldsymbol{\varepsilon}(\boldsymbol{u})=\frac{1}{2}\left(\nabla \boldsymbol{u}+\nabla \boldsymbol{u}^{T}\right) .
$$

$\mu$ denotes the viscosity and $K$ the permeability.

For the analysis it is advantageous to scale the problem. To this end, we rewrite (1) as

$$
-2 K \boldsymbol{A} \boldsymbol{u}+\boldsymbol{u}+\nabla\left(\frac{K}{\mu} p\right)=\frac{K}{\mu} \boldsymbol{f}
$$

By denoting and redefining

$$
t^{2}=2 K, \quad \frac{K}{\mu} p \leftarrow p \text { and } \frac{K}{\mu} \boldsymbol{f} \leftarrow \boldsymbol{f},
$$

we get the scaled version of the Brinkman equations; find $\boldsymbol{u}$ and $p$ such that

$$
\begin{aligned}
-t^{2} \boldsymbol{A} \boldsymbol{u}+\boldsymbol{u}+\nabla p & =\boldsymbol{f} \text { in } \Omega, \\
\operatorname{div} \boldsymbol{u} & =g \text { in } \Omega,
\end{aligned}
$$

where the parameter $0 \leq t \leq C$. For $t=0$ we have the Darcy equations, for which we consider the natural boundary condition

$$
\left.\boldsymbol{u} \cdot \boldsymbol{n}\right|_{\partial \Omega}=0
$$


For $t>0$ we have Dirichlet boundary conditions

$$
\left.\boldsymbol{u}\right|_{\partial \Omega}=\mathbf{0} .
$$

For compatibility, assume $g \in L_{0}^{2}(\Omega)$ and to get a unique pressure assume also $p \in L_{0}^{2}(\Omega)$. When $t \approx 1$ the problem is a standard Stokes problem. For "small" $t$ the problem is a singular perturbation of the Darcy equations. In the analysis natural norm for the velocity is

$$
\|\boldsymbol{v}\|_{t}^{2}=t^{2}\|\boldsymbol{\varepsilon}(\boldsymbol{v})\|_{0}^{2}+\|\boldsymbol{v}\|_{0}^{2}
$$

Hence, for $t=0$ the space for the velocity is $\left[L^{2}(\Omega)\right]^{N}$, and for $t>0$ (by Korn's inequatity) $\left[H_{0}(\Omega)\right]^{N}$. By defining

$$
b(\boldsymbol{v}, q)= \begin{cases}-(\operatorname{div} \boldsymbol{v}, q) & \text { for } t>0 \\ (\boldsymbol{v}, \nabla q) & \text { for } t=0\end{cases}
$$

the norm for the pressure is

$$
\|q\| \|_{t}=\sup _{\boldsymbol{v} \in \boldsymbol{V}} \frac{b(\boldsymbol{v}, q)}{\|\boldsymbol{v}\|_{t}}
$$

and the solution space is

$$
Q=\left\{q \in L_{0}^{2}(\Omega) \mid\|q\|_{t}<\infty\right\} .
$$

Note that for $t=0$ we have

$$
\mid\|q\|_{t} \equiv\|\nabla q\|_{0}
$$

whereas for $0<t \leq C$ the Babuška-Brezzi inequality yields

$$
C_{1}\|q\|_{0} \leq\|\| q\|\|_{t} \leq C_{2} t^{-1}\|q\|_{0} .
$$

Defining the bilinear forms

$$
\begin{gathered}
a(\boldsymbol{u}, \boldsymbol{v})=t^{2}(\boldsymbol{\varepsilon}(\boldsymbol{u}), \boldsymbol{\varepsilon}(\boldsymbol{v}))+(\boldsymbol{u}, \boldsymbol{v}), \\
\mathcal{B}(\boldsymbol{u}, p ; \boldsymbol{v}, q)=a(\boldsymbol{u}, \boldsymbol{v})+b(\boldsymbol{v}, p)+b(\boldsymbol{u}, q),
\end{gathered}
$$

and the linear functional

$$
\mathcal{L}(\boldsymbol{v}, q)=(\boldsymbol{f}, \boldsymbol{v})-(g, q) .
$$

The weak formulation of the problem is then: Find $(\boldsymbol{u}, p) \in \boldsymbol{V} \times Q$ such that

$$
\mathcal{B}(\boldsymbol{u}, p ; \boldsymbol{v}, q)=\mathcal{L}(\boldsymbol{v}, q) \quad \forall(\boldsymbol{v}, q) \in \boldsymbol{V} \times Q .
$$

This is a saddle point problem and Brezzi's conditions imply the stability

$$
\sup _{(\boldsymbol{v}, q) \in \boldsymbol{V} \times Q} \frac{\mathcal{B}(\boldsymbol{w}, r ; \boldsymbol{v}, q)}{\|\boldsymbol{v}\|_{t}+\mid\|q\|_{t}} \geq C\left(\|\boldsymbol{w}\|_{t}+\|\| r \|\left.\right|_{t}\right) \quad \forall(\boldsymbol{w}, r) \in \boldsymbol{V} \times Q
$$

by which the solution is unique. 


\section{$3 \quad$ Finite elements and error estimates}

The fact that the Brinkman model covers a whole range of problems, from Darcy to Stokes, has some consequences. For the Darcy problem a balanced method uses $P_{k}-P_{k-1}$ polynomials for the pressure and velocity, respectively. For the pure Stokes problem (with $t \approx 1$ ) it is the opposite, $P_{k}$ for the velocity and $P_{k-1}$ for the pressure. Hence, to obtain a method good for all values of $t$ it seems natural to use equal order interpolation. Families of this kind are analyzed in our paper [15]. Here we recall the results and also show the results for the well-known Taylor-Hood family of Stokes element.

We assume a partitioning $\mathcal{C}_{h}$ of the domain $\Omega$ into simplices. With $K \in \mathcal{C}_{h}$ we denote an element of the partitioning, and the maximum size of $K \in \mathcal{C}_{h}$ is denoted by $h$. With $\Gamma_{h}$ we denote the boundary edges of the partitioning.

In the following the discrete counterpart of the pressure norm (11) is utilized;

$$
\|\| q\left\|_{t, h}^{2}=\sum_{K \in \mathcal{C}_{h}} \frac{h_{K}^{2}}{t^{2}+h_{K}^{2}}\right\| \nabla q \|_{0, K}^{2} .
$$

This norm has the advantage that it can be explicitly computed.

\subsection{The family generalizing the MINI element}

For this family, generalizing the well-known MINI element of Arnold, Brezzi and Fortin [4]. The finite element spaces are

$$
\begin{aligned}
& \boldsymbol{V}_{h}=\left\{\boldsymbol{v} \in[C(\Omega)]^{N} \cap \boldsymbol{V}|\boldsymbol{v}|_{K} \in\left[P_{k}(K) \cup B_{k+N}(K)\right]^{N}\right\}, \\
& Q_{h}=\left\{q \in C(\Omega) \cap L_{0}^{2}(\Omega)|q|_{K} \in P_{k}(K)\right\},
\end{aligned}
$$

where $P_{k}(K)$ denotes the polynomials of degree $k$ and $B_{k+N}(K)=P_{k+N}(K) \cap$ $H_{0}^{1}(K)$ are the bubbles of degree $k+N$.

The finite element formulation is: find $\left(\boldsymbol{u}_{h}, p_{h}\right) \in \boldsymbol{V}_{h} \times Q_{h}$ such that

$$
\mathcal{B}\left(\boldsymbol{u}_{h}, p_{h} ; \boldsymbol{v}, q\right)=\mathcal{L}(\boldsymbol{v}, q) \quad \forall(\boldsymbol{v}, q) \in \boldsymbol{V}_{h} \times Q_{h} .
$$

The stability is shown in [15]:

Theorem 3.1. There is a constant $C>0$ such that

$$
\sup _{(\boldsymbol{v}, q) \in \boldsymbol{V}_{h} \times Q_{h}} \frac{\mathcal{B}(\boldsymbol{w}, r ; \boldsymbol{v}, q)}{\|\boldsymbol{v}\|_{t}+\mid\|q\|_{t, h}} \geq C\left(\|\boldsymbol{w}\|_{t}+\|\| r \|_{t, h}\right) \quad \forall(\boldsymbol{w}, r) \in \boldsymbol{V}_{h} \times Q_{h} .
$$

The stability gives the quasioptimal a priori result [15]:

Theorem 3.2. There exists $C>0$ such that

$$
\begin{aligned}
\left\|\boldsymbol{u}-\boldsymbol{u}_{h}\right\|_{t}+\left|\left\|p-p_{h}\right\|\right|_{t, h} \leq & C\left(\inf _{\boldsymbol{v} \in \boldsymbol{V}_{h}}\left\{\|\boldsymbol{u}-\boldsymbol{v}\|_{t}+t\left(\sum_{K \in \mathcal{C}_{h}} h_{K}^{-2}\|\boldsymbol{u}-\boldsymbol{v}\|_{0, K}^{2}\right)^{1 / 2}\right\}\right. \\
& \left.+\inf _{q \in Q_{h}}\left\{\left.\|p-q\|\right|_{t, h}+\mid\|p-q\|_{t}\right\}\right) .
\end{aligned}
$$


We have also proved an a posteriori estimate. Since this is the same for all methods considered we give it in Section 3.4 below. Due to the boundary layer and corner singularities, the solution to the equations is never smooth. Nevertheless it is instructive to look at the error estimate assuming a smooth solution and a quasiuniform mesh. In this case we get the estimate

$$
\left\|\boldsymbol{u}-\boldsymbol{u}_{h}\right\|_{t}+\left|\left\|p-p_{h}\right\|\right|_{t, h} \leq C\left((t+h) h^{k}\|\boldsymbol{u}\|_{k+1}+(t+h)^{-1} h^{k+1}\|p\|_{k+1}\right) .
$$

Hence, we get a uniform convergence (with respect to $t$ ) of $\mathcal{O}\left(h^{k}\right)$.

\subsection{Stabilized methods}

The linear stabilized method was introduced by Brezzi and Pitkäranta [9] and then generalized by Hughes and Franca [14]. In [15] we analyze the method using the techniques developed in $[12,11]$.

The method uses pure piecewise polynomials of equal degree:

$$
\begin{aligned}
& \boldsymbol{V}_{h}=\left\{\boldsymbol{v} \in[C(\Omega)]^{N} \cap \boldsymbol{V}|\boldsymbol{v}|_{K} \in\left[P_{k}(K)\right]^{N}\right\}, \\
& Q_{h}=\left\{q \in C(\Omega) \cap L_{0}^{2}(\Omega)|q|_{K} \in P_{k}(K)\right\} .
\end{aligned}
$$

The stabilized method is then: Find $\left(\boldsymbol{u}_{h}, p_{h}\right) \in \boldsymbol{V}_{h} \times Q_{h}$ such that

$$
\mathcal{B}_{h}\left(\boldsymbol{u}_{h}, p_{h} ; \boldsymbol{v}, q\right)=\mathcal{L}_{h}(\boldsymbol{v}, q) \quad \forall(\boldsymbol{v}, q) \in \boldsymbol{V}_{h} \times Q_{h},
$$

with

$$
\begin{aligned}
\mathcal{B}_{h}\left(\boldsymbol{u}_{h}, p_{h} ; \boldsymbol{v}, q\right)= & \mathcal{B}\left(\boldsymbol{u}_{h}, p_{h} ; \boldsymbol{v}, q\right) \\
& -\alpha \sum_{K \in \mathcal{C}_{h}} \frac{h_{K}^{2}}{t^{2}+h_{K}^{2}}\left(t^{2} \boldsymbol{A} \boldsymbol{u}_{h}-\boldsymbol{u}_{h}-\nabla p_{h}, t^{2} \boldsymbol{A} \boldsymbol{v}-\boldsymbol{v}-\nabla q\right)_{K}
\end{aligned}
$$

and

$$
\mathcal{L}_{h}(\boldsymbol{v}, q)=\mathcal{L}(\boldsymbol{v}, q)-\alpha \sum_{K \in \mathcal{C}_{h}} \frac{h_{K}^{2}}{t^{2}+h_{K}^{2}}\left(\boldsymbol{f}, t^{2} \boldsymbol{A} \boldsymbol{v}-\boldsymbol{v}-\nabla q\right)_{K},
$$

with a parameter $\alpha>0$. For consistency, assume

$$
t^{2} \boldsymbol{A} \boldsymbol{u}-\boldsymbol{u}-\nabla p=\boldsymbol{f} \in\left[L^{2}(\Omega)\right]^{2} .
$$

Then it holds

$$
\mathcal{B}_{h}\left(\boldsymbol{u}-\boldsymbol{u}_{h}, p-p_{h} ; \boldsymbol{v}, q\right)=0 \quad \forall(\boldsymbol{v}, q) \in \boldsymbol{V}_{h} \times Q_{h}
$$

By $C_{I}$ we denote the constant in the following inverse inequality

$$
h_{K}^{2}\|\boldsymbol{A} \boldsymbol{w}\|_{0, K}^{2} \leq C_{I}\|\nabla \boldsymbol{w}\|_{0, K}^{2} \quad \forall \boldsymbol{w} \in\left[P_{k}(K)\right]^{N} .
$$

The stability result is: 
Theorem 3.3. For $0 \leq \alpha \leq \min \left\{1 /\left(2 C_{I}\right), 1 / 2\right\}$ there exists $C>0$ such that

$$
\sup _{(\boldsymbol{v}, q) \in \boldsymbol{V}_{h} \times Q_{h}} \frac{\mathcal{B}_{h}(\boldsymbol{w}, r ; \boldsymbol{v}, q)}{\|\boldsymbol{v}\|_{t}+\mid\|q\|_{t, h}} \geq C\left(\|\boldsymbol{w}\|_{t}+|\|r\||_{t, h}\right) \quad \forall(\boldsymbol{w}, r) \in \boldsymbol{V}_{h} \times Q_{h} .
$$

Again we have a quasioptimal error estimate.

Theorem 3.4. Assume that $0<\alpha<\min \left\{1 /\left(2 C_{I}\right), 1 / 2\right\}$. Then it holds

$$
\begin{aligned}
& \left\|\boldsymbol{u}-\boldsymbol{u}_{h}\right\|_{t}+\left|\left\|p-p_{h}\right\|\right|_{t, h} \\
& \leq C \inf _{(\boldsymbol{v}, q) \in \boldsymbol{V}_{h} \times Q_{h}}\left\{\|\boldsymbol{u}-\boldsymbol{v}\|_{t}+t\left(\sum_{K \in \mathcal{C}_{h}} h_{K}^{-2}\|\boldsymbol{u}-\boldsymbol{v}\|_{0, K}^{2}\right)^{1 / 2}\right. \\
& \quad+|\|p-q\||_{t, h}+|\|p-q\||_{t} \\
& \left.\quad+\left(\sum_{K \in \mathcal{C}_{h}} \frac{h_{K}^{2}}{t^{2}+h_{K}^{2}}\left\|t^{2} \boldsymbol{A} \boldsymbol{v}-\boldsymbol{v}-\nabla q+\boldsymbol{f}\right\|_{0, K}^{2}\right)^{1 / 2}\right\} .
\end{aligned}
$$

We have written the estimate in this form in order to emphasize that one does not have to assume that $t^{2} \boldsymbol{A u} \in\left[L^{2}(\Omega)\right]^{N}$ and $\nabla p \in\left[L^{2}(\Omega)\right]^{N}$, only that $\boldsymbol{f} \in\left[L^{2}(\Omega)\right]^{N}$.

For a smooth solution and a quasiuniform mesh we again get the uniform $\mathcal{O}\left(h^{k}\right)$ estimate $(26)$.

\subsection{The Taylor-Hood family}

The third method to be considered is the Taylor-Hood family with the finite element subspaces

$$
\begin{aligned}
\boldsymbol{V}_{h} & =\left\{\boldsymbol{v} \in[C(\Omega)]^{N} \cap \boldsymbol{V}|\boldsymbol{v}|_{K} \in\left[P_{k+1}(K)\right]^{N}\right\}, \\
Q_{h} & =\left\{q \in C(\Omega) \cap L_{0}^{2}(\Omega)|q|_{K} \in P_{k}(K)\right\} .
\end{aligned}
$$

The finite element formulation is: find $\left(\boldsymbol{u}_{h}, p_{h}\right) \in \boldsymbol{V}_{h} \times Q_{h}$ such that

$$
\mathcal{B}\left(\boldsymbol{u}_{h}, p_{h} ; \boldsymbol{v}, q\right)=\mathcal{L}(\boldsymbol{v}, q) \quad \forall(\boldsymbol{v}, q) \in \boldsymbol{V}_{h} \times Q_{h} .
$$

For the Stokes problem $(t \approx 1)$ this method has been proved to be optimal both in two and three space dimensions $[5,22,21,8,23,6,7,10]$. By established techniques the analysis can be carried over to the present case and Theorems 3.1 and 3.2 are valid.

For this family the assumption of a quasiuniform mesh and a smooth solution gives the estimate

$$
\left\|\boldsymbol{u}-\boldsymbol{u}_{h}\right\|_{t}+\left|\left\|p-p_{h}\right\|\right|_{t, h} \leq C\left((t+h) h^{k+1}\|\boldsymbol{u}\|_{k+2}+(t+h)^{-1} h^{k+1}\|p\|_{k+1}\right) .
$$

From here we see that also for this method we a uniform convergence of $\mathcal{O}\left(h^{k}\right)$. Only for the Stokes limit with $t \approx 1$ we have a $\mathcal{O}\left(h^{k+1}\right)$ convergence rate. In the Darcy limit $t=0$ the two terms are not in balance, $\mathcal{O}\left(h^{k+2}\right)$ and $\mathcal{O}\left(h^{k}\right)$, respectively. 


\subsection{The a posteriori estimate}

In this section a residual based a posteriori estimator is introduced. The a posteriori results hold for all the elements considered in the previous section. For the derivation and analysis of the estimator we refer to [15].

The elementwise estimator is

$$
\begin{aligned}
E_{K}\left(\boldsymbol{u}_{h}, p_{h}\right)^{2}= & \frac{h_{K}^{2}}{t^{2}+h_{K}^{2}}\left\|t^{2} \boldsymbol{A} \boldsymbol{u}_{h}-\boldsymbol{u}_{h}-\nabla p_{h}+\boldsymbol{f}\right\|_{0, K}^{2} \\
& +\left(t^{2}+h_{K}^{2}\right)\left\|\operatorname{div} \boldsymbol{u}_{h}-g\right\|_{0, K}^{2} \\
& +\frac{h_{K}}{t^{2}+h_{K}^{2}}\left\|\llbracket t^{2} \varepsilon_{n}\left(\boldsymbol{u}_{h}\right) \rrbracket\right\|_{0, \partial K \backslash \partial \Omega}^{2}+\frac{t^{2}+h_{K}^{2}}{h_{K}}\left\|\boldsymbol{u}_{h} \cdot \boldsymbol{n}\right\|_{0, \partial K \cap \partial \Omega}^{2}
\end{aligned}
$$

and the global estimator is

$$
\eta=\left(\sum_{K \in \mathcal{C}_{h}} E_{K}\left(\boldsymbol{u}_{h}, p_{h}\right)^{2}\right)^{1 / 2} .
$$

Here $\varepsilon_{n}(\cdot)$ denotes the normal derivative and $\llbracket \cdot \rrbracket$ is the jump. Notice that the last term in (41) vanishes for $t>0$.

In the limit $t=0$ (or as $t<h$ ) the a posteriori estimator becomes

$$
\begin{aligned}
E_{K}\left(\boldsymbol{u}_{h}, p_{h}\right)^{2} \approx & \left\|\boldsymbol{u}_{h}+\nabla p_{h}-\boldsymbol{f}\right\|_{0, K}^{2} \\
& +h_{K}^{2}\left\|\operatorname{div} \boldsymbol{u}_{h}-g\right\|_{0, K}^{2}+h_{E}\left\|\boldsymbol{u}_{h} \cdot \boldsymbol{n}\right\|_{0, \partial K \cap \partial \Omega}^{2}
\end{aligned}
$$

which is the estimator for the Darcy problem. On the other hand, if $t \geq C>$ 0 , the estimator can be expressed as

$$
\begin{aligned}
E_{K}\left(\boldsymbol{u}_{h}, p_{h}\right)^{2} \approx & h_{K}^{2}\left\|t^{2} \boldsymbol{A} \boldsymbol{u}_{h}-\boldsymbol{u}_{h}-\nabla p_{h}+\boldsymbol{f}\right\|_{0, K}^{2}+\left\|\operatorname{div} \boldsymbol{u}_{h}-g\right\|_{0, K}^{2} \\
& +h_{E}\left\|\llbracket t^{2} \boldsymbol{\varepsilon}_{n}\left(\boldsymbol{u}_{h}\right) \rrbracket\right\|_{0, \partial K \backslash \partial \Omega}^{2},
\end{aligned}
$$

which is the standard Stokes estimator.

Under a saturation assumption we are able prove the following theorem[15].

Theorem 3.5. There exists $C>0$ such that

$$
\left\|\boldsymbol{u}-\boldsymbol{u}_{h}\right\|_{t}+\mid\left\|p-p_{h}\right\|_{t, h} \leq C \eta
$$

The a posteriori estimator is also a lower bound to the error. In this sense the estimator is sharp.

Theorem 3.6. There exist $C>0$ such that

$$
\begin{aligned}
C \eta^{2} \leq & \left\|\boldsymbol{u}-\boldsymbol{u}_{h}\right\|_{t}^{2}+\mid\left\|p-p_{h}\right\|_{t, h}^{2} \\
& +\sum_{K \in \mathcal{C}_{h}}\left(\frac{h_{K}^{2}}{t^{2}+h_{K}^{2}}\left\|\boldsymbol{f}-\boldsymbol{f}_{h}\right\|_{0, K}^{2}+\left(t^{2}+h_{K}^{2}\right)\left\|g-g_{h}\right\|_{0, K}^{2}\right),
\end{aligned}
$$

with $\boldsymbol{f}_{h} \in \boldsymbol{V}_{h}$ and $g_{h} \in Q_{h}$ being interpolants to $\boldsymbol{f}$ and $g$, respectively. 


\subsection{Nitsche's method for imposing boundary conditions}

In this section the modified method of imposing the Dirichlet boundary conditions in a weak sense using the technique of Nitsche $[19,15,16]$ is outlined. With this, we obtain formulations that use the same finite element spaces both for $t>0$ and in the limit $t=0$. Recall that in the methods above, the boundary conditions disappear from the definition of $\boldsymbol{V}_{h}$ in the limit $t=0$.

For notational convenience we have above assumed homogeneous boundary conditions. Since Nitsche's method is not so standard, we will here describe the method assuming nonhomogeneous boundary conditions, viz.

$$
\left\{\begin{array}{l}
\left.\boldsymbol{u}\right|_{\partial \Omega}=\boldsymbol{u}_{\Gamma} \text { for } t>0, \\
\left.\boldsymbol{u} \cdot \boldsymbol{n}\right|_{\partial \Omega}=\boldsymbol{u}_{\Gamma} \cdot \boldsymbol{n} \text { for } t=0 .
\end{array}\right.
$$

For Nitsche's method the velocity space is modified by removing the Dirichlet boundary conditions from $\boldsymbol{V}_{h}$ even for $t>0$, i.e $\boldsymbol{v} \in[C(\Omega)]^{N} \cap \boldsymbol{V}$ is replaced by $\boldsymbol{v} \in[C(\Omega)]^{N}$ in (21), (27) and (37). The nonhomogeneouity of the boundary conditions implies that the term

$$
\left\langle\boldsymbol{u}_{\Gamma} \cdot \boldsymbol{n}, q\right\rangle
$$

has to be included in the right hand side of the weak forms.

The discrete variational formulations are modified by changing the bilinear form $a(\cdot, \cdot)$ to

$$
\begin{aligned}
a_{h}(\boldsymbol{u}, \boldsymbol{v})= & a(\boldsymbol{u}, \boldsymbol{v}) \\
& +t^{2} \sum_{E \in \Gamma_{h}}\left(-\left\langle\varepsilon_{n}(\boldsymbol{u}), \boldsymbol{v}\right\rangle_{E}-\left\langle\varepsilon_{n}(\boldsymbol{v}), \boldsymbol{u}\right\rangle_{E}+\gamma h_{E}^{-1}\langle\boldsymbol{u}, \boldsymbol{v}\rangle_{E}\right),
\end{aligned}
$$

where $\Gamma_{h}$ denotes the edges/faces on the boundary $\partial \Omega$. The parameter has to satisfy $\gamma>C_{I}^{\prime}$, where $C_{I}^{\prime}$ is the constant in the discrete trace inequality

$$
h_{E}\left\|\varepsilon_{n}(\boldsymbol{v})\right\|_{0, \partial K}^{2} \leq\left. C_{I}^{\prime}\|\nabla \boldsymbol{v}\|_{0, K}^{2} \quad \forall \boldsymbol{v} \in \boldsymbol{V}_{h}\right|_{K} .
$$

Then the ellipticity

$$
a_{h}(\boldsymbol{v}, \boldsymbol{v}) \geq C\|\boldsymbol{v}\|_{t, h}^{2} \quad \forall \boldsymbol{v} \in \boldsymbol{V}_{h}
$$

with

$$
\|\boldsymbol{v}\|_{t, h}^{2}=\|\boldsymbol{v}\|_{t}^{2}+t^{2} \sum_{E \in \Gamma_{h}} h_{E}^{-1}\|\boldsymbol{v}\|_{0, E}^{2}
$$

holds. In addition to (46) the following term has to be added to the right hand side

$$
t^{2} \sum_{E \in \Gamma_{h}}\left(-\left\langle\varepsilon_{n}(\boldsymbol{v}), \boldsymbol{u}_{\Gamma}\right\rangle_{E}+\gamma h_{E}^{-1}\left\langle\boldsymbol{u}_{\Gamma}, \boldsymbol{v}\right\rangle_{E}\right) .
$$


For a posteriori result the elementwise estimator $E_{K}\left(\boldsymbol{u}_{h}, p_{h}\right)^{2}(41)$ is changed by adding the term

$$
\frac{t^{2}}{h_{K}}\left\|\boldsymbol{u}_{h}-\boldsymbol{u}_{\Gamma}\right\|_{0, \partial K \cap \partial \Omega}^{2}
$$

and changing

$$
\frac{t^{2}+h_{K}^{2}}{h_{K}}\left\|\boldsymbol{u}_{h} \cdot \boldsymbol{n}\right\|_{0, \partial K \cap \partial \Omega}^{2} \quad \text { to } \quad \frac{t^{2}+h_{K}^{2}}{h_{K}}\left\|\left(\boldsymbol{u}_{h}-\boldsymbol{u}_{\Gamma}\right) \cdot \boldsymbol{n}\right\|_{0, \partial K \cap \partial \Omega}^{2}
$$

Using the global estimator $\eta(42)$ defined with the above local estimator it holds

$$
C_{1} \eta \leq\left\|\boldsymbol{u}-\boldsymbol{u}_{h}\right\|_{t, h}+\mid\left\|p-p_{h}\right\|_{t, h} \leq C_{2} \eta
$$

\section{Numerical examples}

The finite element methods tested here are the lowest order MINI and TaylorHood elements and stabilized $P_{1}-P_{1}$ and $P_{2}-P_{2}$ methods. In all the computations the residual stabilization parameter is $\alpha=0.4$ for the P1-P1-stab and $\alpha=0.01$ for the P2-P2-stab. The stabilization parameter of Nitsche's method is $\gamma=35$. We use Dirichlet condition for the velocity on all boundaries.

Our first model problem is the L-shape domain with the solution

$$
\begin{aligned}
& p(r, \theta)=r^{\beta} \sin (\beta \theta)+C \quad \text { and } \\
& \boldsymbol{u}=-\nabla p(x, y)=\beta r^{\beta-1}\left(\begin{array}{c}
-\sin (\theta-\beta \theta) \\
\cos (\theta-\beta \theta)
\end{array}\right),
\end{aligned}
$$

where $(r, \theta)$ are the polar coordinates, $\beta>0$ is a parameter and $C$ is a constant such that $p \in L_{0}^{2}(\Omega)$. The smoothness of the solution is $p \in H^{\beta+1}$ and $\boldsymbol{u} \in\left[H^{\beta}\right]^{2}$. In Figure 1 is the error as a function of degrees of freedom for different values of the parameter $t$. The value of the smoothness parameter is $\beta=3.1$. This means that the solution is smooth enough to take the full advantage of the higher order methods P2-P2-stab and Taylor-Hood even in the Stokes problem. All the methods perform as predicted by the theory. Notice the $\mathcal{O}(h)$ rate of convergence of the Taylor-Hood element in the Darcy type problem, that is when the parameter $t$ is small. For more details see equation (40) and the discussion within.

Our next model problem is the Poiseuille flow in a unit square $\Omega=$ $(0,1) \times(0,1)$. The Poiseuille flow is such that $\boldsymbol{f}$ and $g$ vanish, and the linearly decreasing pressure drives the flow. Assume the pressure is $p=-x+\frac{1}{2}$ and that the velocity is zero at the sides $y=0$ and $y=1$. The solution of this problem is $\boldsymbol{u}=(u, 0)^{T}$ where

$$
u= \begin{cases}\left(1+e^{1 / t}-e^{(1-y) / t}-e^{y / t}\right) /\left(1+e^{1 / t}\right) & \text { if } t>0 \\ 1 \quad \text { if } t=0 & \end{cases}
$$


In Figure 2 are some flow profiles of this solution. Notice the steep changes in the solution near the boundaries if the value of the parameter $t$ is small.

Due to the boundary layers the Poiseuille flow problem is ideal for testing the effect of boundary conditions. To this end, the rate of convergence is computed using both the traditional boundary conditions and Nitsche's method. In Figure 3 are the rates of convergence using the MINI element. Since the ratio of the mesh size $h$ and the parameter $t$ is crucial, the results are plotted as a function of $h / t$. For both methods the rate of convergence is slower if $h>t$. For Nitsche's method the rate of convergence is very limited but the error is already much smaller than for the traditional boundary conditions. Also, notice the clear change in the rate of convergence for both the methods in the limit $t \approx h$. This is due to the transition from the sigularly perturbed Darcy problem to the Stokes type problem as the mesh size becomes smaller than $t$. This suggests that numerically the 'limit' for the Brinkman problem being singularly perturbed is $t \approx h$. Similar result holds also for the reaction-diffusion problem [17].

To examine the slow convergence near the Darcy limit in more detail, the convergence of the different components of the energy norm are studied. Since the gradient of the pressure is constant, the velocity part of the error dominates the total error. In Figure 4 are the rates of convergence for different components of the velocity energy norm, namely for the $t\left\|\nabla\left(\boldsymbol{u}-\boldsymbol{u}_{h}\right)\right\|_{0}$-part and the $\left\|\boldsymbol{u}-\boldsymbol{u}_{h}\right\|_{0}$-part. Surprisingly, for the traditional method the $L^{2}$-part dominates the error for $h>t$. Nitsche's method, on the other hand, is able to balance the error between the components for $h>t$.

Above it is studied how the boundary conditions affect the solution in the energy norm. To see what happens in the $L^{\infty}$-norm consider a simplified problem: assume the pressure is already known, then the problem for the $x$-component of the velocity becomes

$$
-t^{2} u^{\prime \prime}(y)+u(y)=1 \quad \text { and } \quad u(0)=u(1)=0 .
$$

Solving this simple 1D problem with the finite element method gives good insight in what happens in $L^{\infty}$-norm. In Figure 5 we have the solutions to the above problem using both Nitsche's and the traditional method. The velocity energy norm has two major components, namely $t\left\|\nabla\left(\boldsymbol{u}-\boldsymbol{u}_{h}\right)\right\|_{0}$ and $\left\|\boldsymbol{u}-\boldsymbol{u}_{h}\right\|$. For small values of $t$ the weight of the gradient error is considerably smaller hence Nitsche's method relaxes the boundary conditions to reduce the dominating $L^{2}$-error. The traditional method does not have degrees of freedom on the boundary and cannot relax the boundary conditions. As the mesh size $h$ becomes smaller both methods give the same solution. Figure 5 also shows how the boundary conditions transform naturally from the Stokes condition $\boldsymbol{u}=0$ to Darcy condition $\boldsymbol{u} \cdot \boldsymbol{n}=0$ in Nitsche's method. In [13] similar results are observed.

Lastly we test how the proposed a posteriori estimator (42) works in adaptive refinement. The implementation here is to refine all the elements whose elementwise indicator is larger than (or equal to) the mean value of the elementwise indicators. In Figure 6 is the error in the energy norm with 
respect to the degrees of freedom using both the adaptive and the uniform refinement. Initially the parameter $t$ is small compared to the mesh size $h$, hence the convergence is very limited using uniform refinement. However, the adaptive refinement detects the source of error, see Figure 7, and increases the rate of convergence substantially.

\section{References}

[1] G. Allaire. Homogenization of the Navier-Stokes equations in open sets perforated with tiny holes. I. abstract framework, a volume distribution of holes. Arch. Rational Mech. Anal., 113(3):209-259, 1990.

[2] G. Allaire. Homogenization of the Navier-Stokes equations in open sets perforated with tiny holes. II. Noncritical sizes of the holes for a volume distribution and a surface distribution of holes. Arch. Rational Mech. Anal., 113(3):261298, 1990.

[3] T. Arbogast and H. L. Lehr. Homogenization of a Darcy-Stokes system modeling vuggy porous media. Comput. Geosci., 10(3):291-302, 2006.

[4] D. N. Arnold, F. Brezzi, and M. Fortin. A stable finite element for the Stokes equations. Calcolo, 21(4):337-344 (1985), 1984.

[5] M. Bercovier and O. Pironneau. Error estimates for finite element method solution of the Stokes problem in the primitive variables. Numer. Math., $33(2): 211-224,1979$

[6] D. Boffi. Stability of higher order triangular Hood-Taylor methods for the stationary Stokes equations. Math. Models Methods Appl. Sci., 4(2):223-235, 1994 .

[7] D. Boffi. Three-dimensional finite element methods for the Stokes problem. SIAM J. Numer. Anal., 34(2):664-670, 1997.

[8] F. Brezzi and R. S. Falk. Stability of higher-order Hood-Taylor methods. SIAM J. Numer. Anal., 28(3):581-590, 1991.

[9] F. Brezzi and J. Pitkäranta. On the stabilization of finite element approximations of the Stokes equations. In Efficient solutions of elliptic systems (Kiel, 1984), volume 10 of Notes Numer. Fluid Mech., pages 11-19. Vieweg, Braunschweig, 1984.

[10] Richard S. Falk. A Fortin operator for two-dimensional Taylor-Hood elements. M2AN Math. Model. Numer. Anal., 42(3):411-424, 2008.

[11] L.P. Franca, T.J.R. Hughes, and R. Stenberg. Stabilized finite element methods. In M. Gunzburger and R.A. Nicolaides, editors, Incompressible Computational Fluid Dynamics, chapter 4, pages 87-107. Cambridge University Press, 1993.

[12] L.P. Franca and R. Stenberg. Error analysis of Galerkin least squares methods for the elasticity equations. SIAM J. Numer. Anal., 28(6):1680-1697, 1991. 
[13] P. Hansbo and M. Juntunen. Weakly imposed dirichlet boundary conditions for the brinkman model of porous media flow. Appl. Numer. Math., 59:1274$1289,2009$.

[14] T. J. R. Hughes and L. P. Franca. A new finite element formulation for computational fluid dynamics. VII. The Stokes problem with various wellposed boundary conditions: symmetric formulations that converge for all velocity/pressure spaces. Comput. Methods Appl. Mech. Engrg., 65(1):85-96, 1987.

[15] M. Juntunen and R. Stenberg. Analysis of finite element methods for the Brinkman problem. Helsinki University of Technology Institute of Mathematics Research Report A 557, 2009.

[16] M. Juntunen and R. Stenberg. Nitsche's method for general boundary conditions. Math. Comp., 2008.

[17] M. Juntunen and R. Stenberg. A residual based a posteriori estimator for the reaction-diffusion problem. C. R. Acad. Sci. Paris, Ser. I*** (***), Available online, 2009.

[18] T. Lévy. Loi de Darcy ou loi de Brinkman? C. R. Acad. Sci. Paris Sér. II Méc. Phys. Chim. Sci. Univers Sci. Terre, 292(12):871-874, Erratum (17):1239, 1981.

[19] J.A. Nitsche. Über ein Variationsprinzip zur Lösung von Dirichlet-Problemen bei Verwendung von Teilräumen, die keinen Randbedingungen unterworfen sind. Abhandlungen aus dem Mathematischen Seminar der Universität Hamburg, 36:9-15, 1970/71.

[20] K. R. Rajagopal. On a hierarchy of approximate models for flows of incompressible fluids through porous solids. Math. Models Methods Appl. Sci., $17(2): 215-252,2007$.

[21] L. R. Scott and M. Vogelius. Norm estimates for a maximal right inverse of the divergence operator in spaces of piecewise polynomials. RAIRO Modél. Math. Anal. Numér., 19(1):111-143, 1985.

[22] R. Stenberg. On some three-dimensional finite elements for incompressible media. Comput. Methods Appl. Mech. Engrg., 63(3):261-269, 1987.

[23] R. Stenberg. Error analysis of some finite element methods for the Stokes problem. Math. Comp., 54:495-508, 1990. 

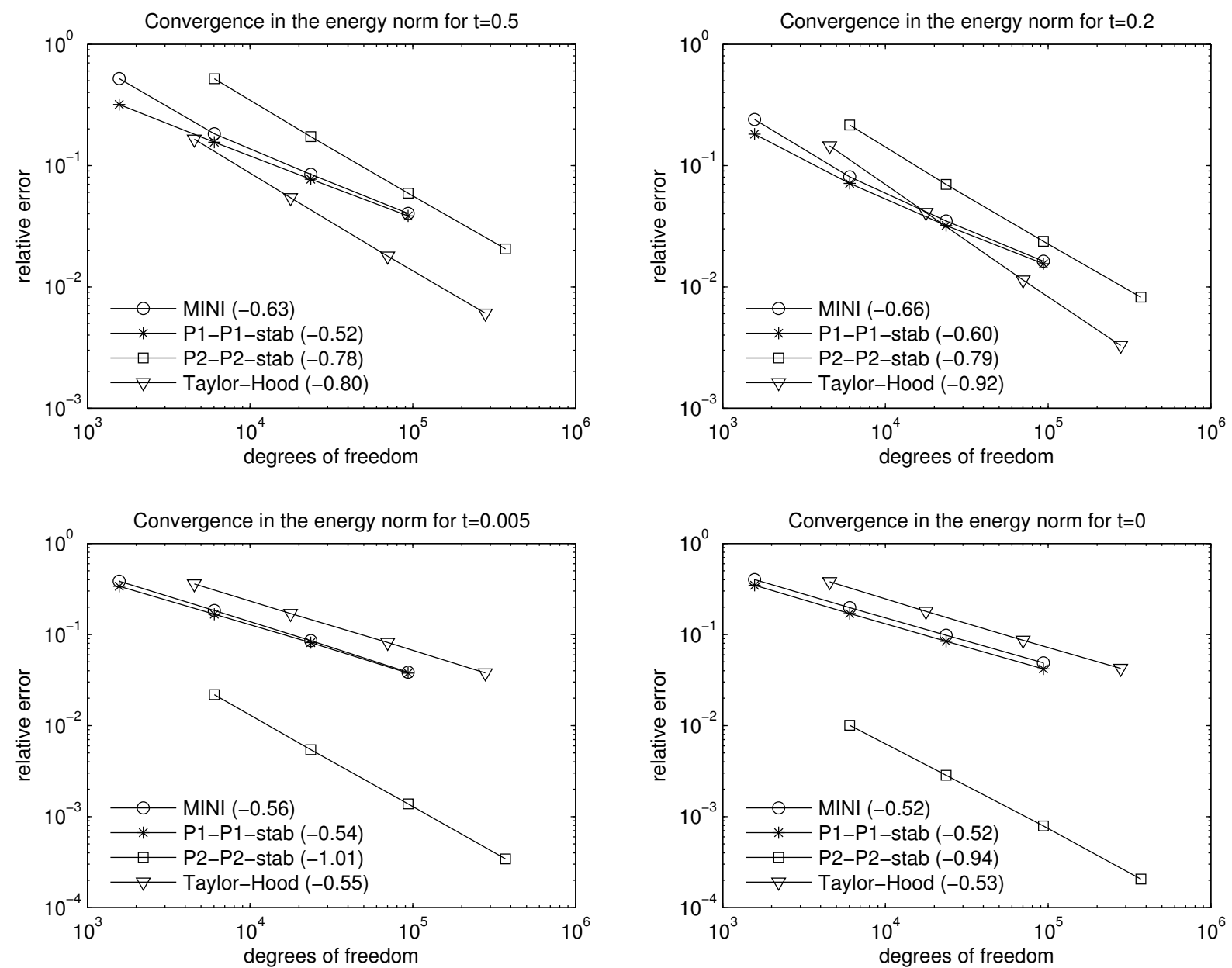

Figure 1: Convergence of the finite element solutions in the energy norm using uniform refinement. The value in the brackets is the average rate of convergence; values -0.5 and -1.0 correspond to $\mathcal{O}(h)$ and $\mathcal{O}\left(h^{2}\right)$ rates of convergence. On the top row the problem is of the Stokes type and on the bottom row of the Darcy type. Notice the $\mathcal{O}(h)$ rate of convergence of the Taylor-Hood element in the Darcy type problem even though the solution is smooth. 


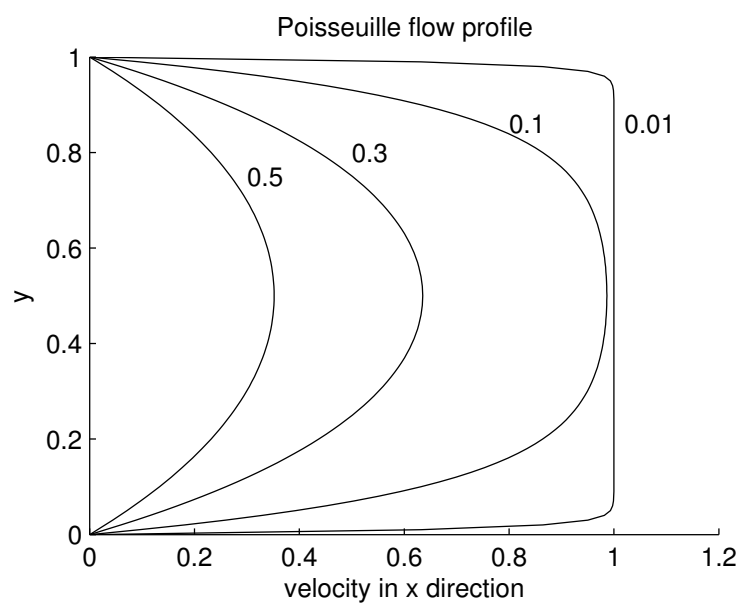

Figure 2: The Poisseuille flow profile for $t$ equal to $0.5,0.3,0.1$ and 0.01 .
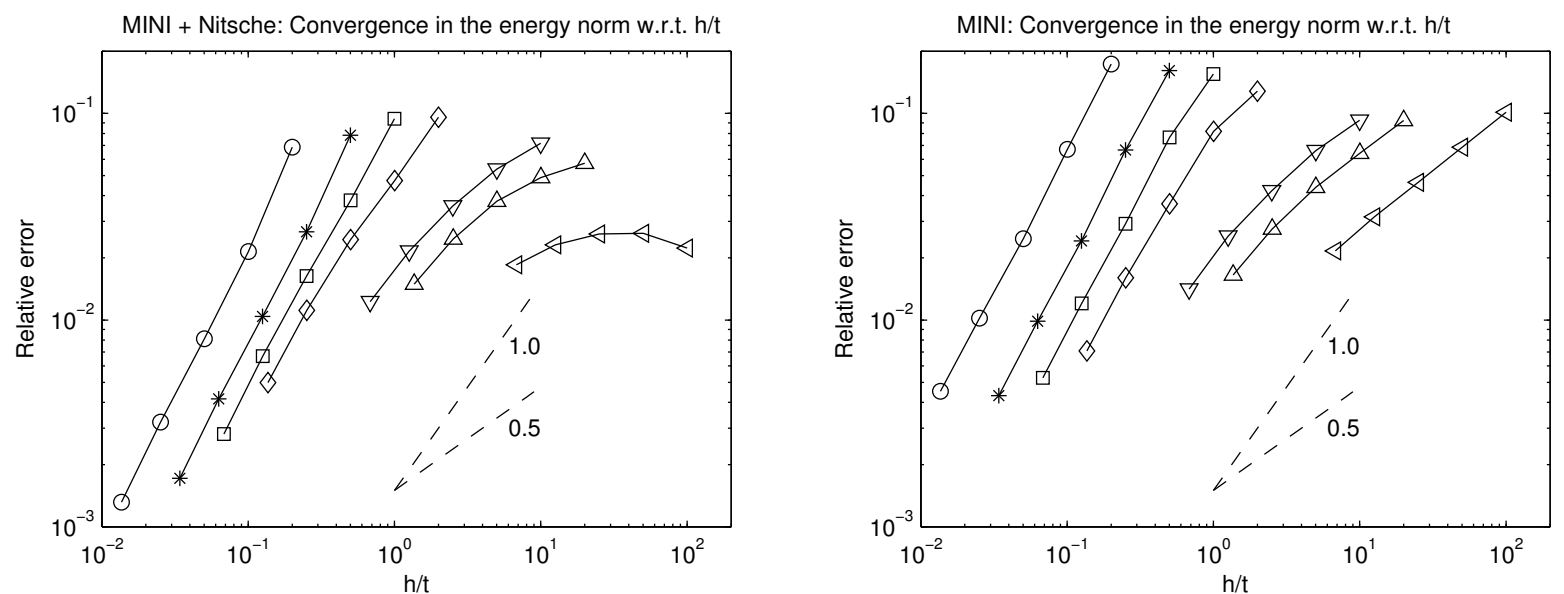

Figure 3: Convergence of the finite element solution in the energy norm w.r.t $h / t$ using uniform refinement; on the left using Nitsche's method and on the right using the traditional boundary conditions. Dashed lines are reference slopes of $\mathcal{O}(\sqrt{h})$ and $\mathcal{O}(h)$ convergence. Notice the slower convergence of both methods if $h>t$. 

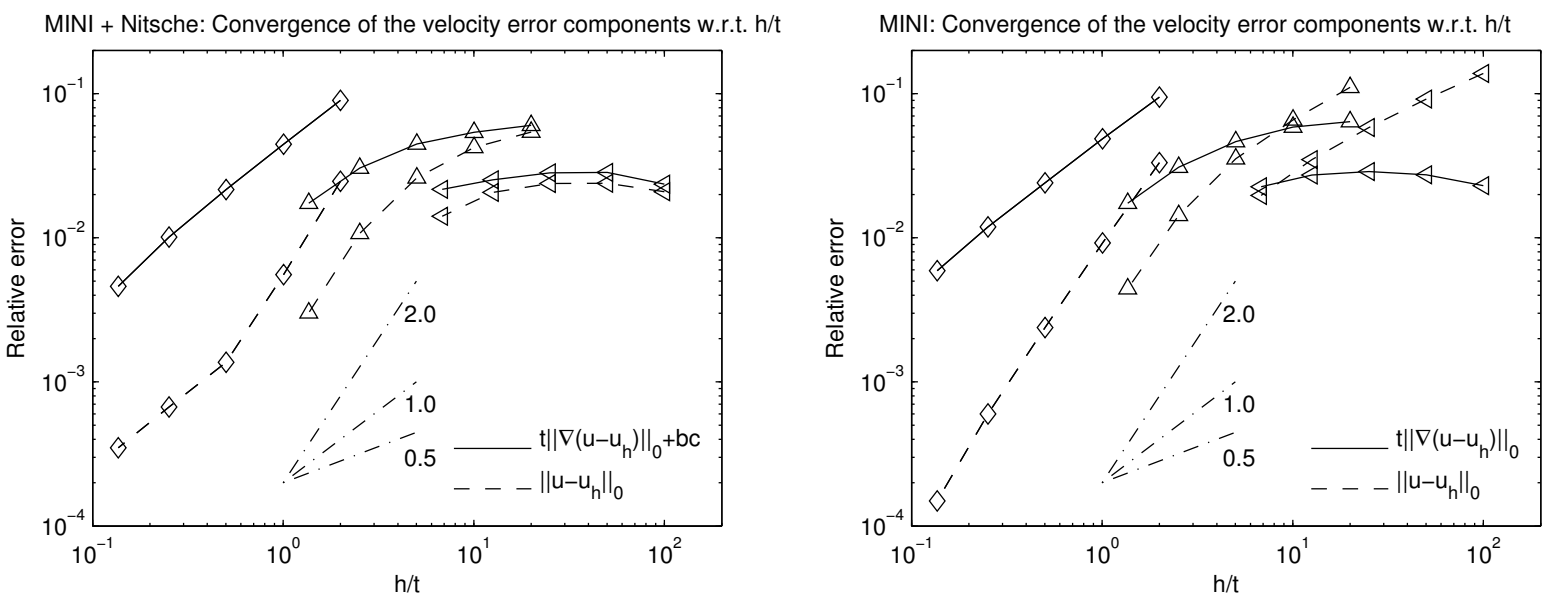

Figure 4: Convergence of the two components of the velocity energy norm w.r.t $h / t$ using uniform refinement; on the left with Nitsche's method and on the right with the traditional boundary conditions. Notice that the $L^{2}$ component of the error dominates for $h>t$ in the traditional method whereas Nitsce's method is able to balance the error between the components. 

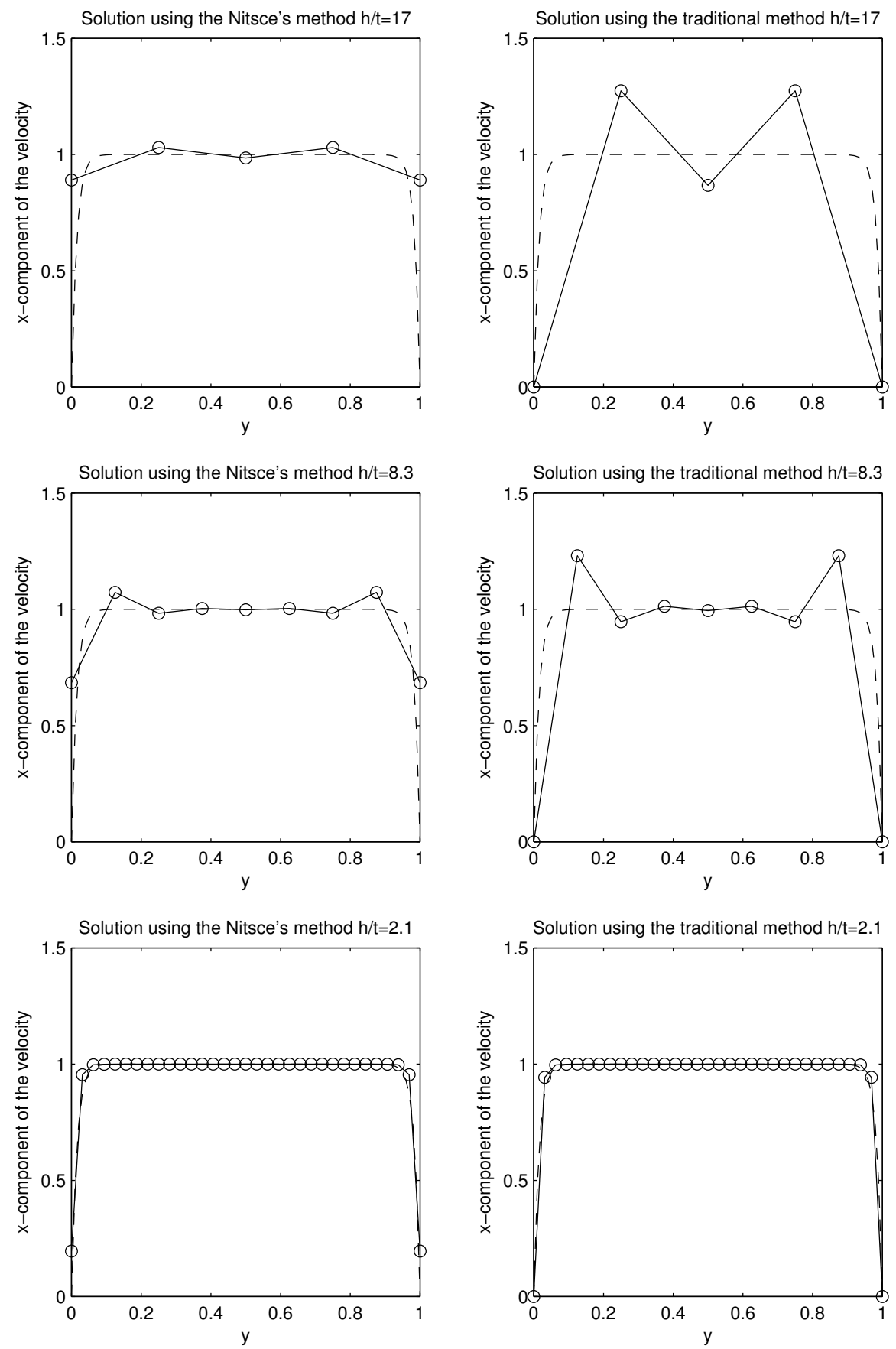

Figure 5: Finite element solutions to the problem (56); on the left with Nitsche's and on the right with the traditional method. Dashed line is the exact solution. The $h / t$ ratio is in the title. Notice how Nitsche's method relaxes the boundary conditions if $h>t$. 

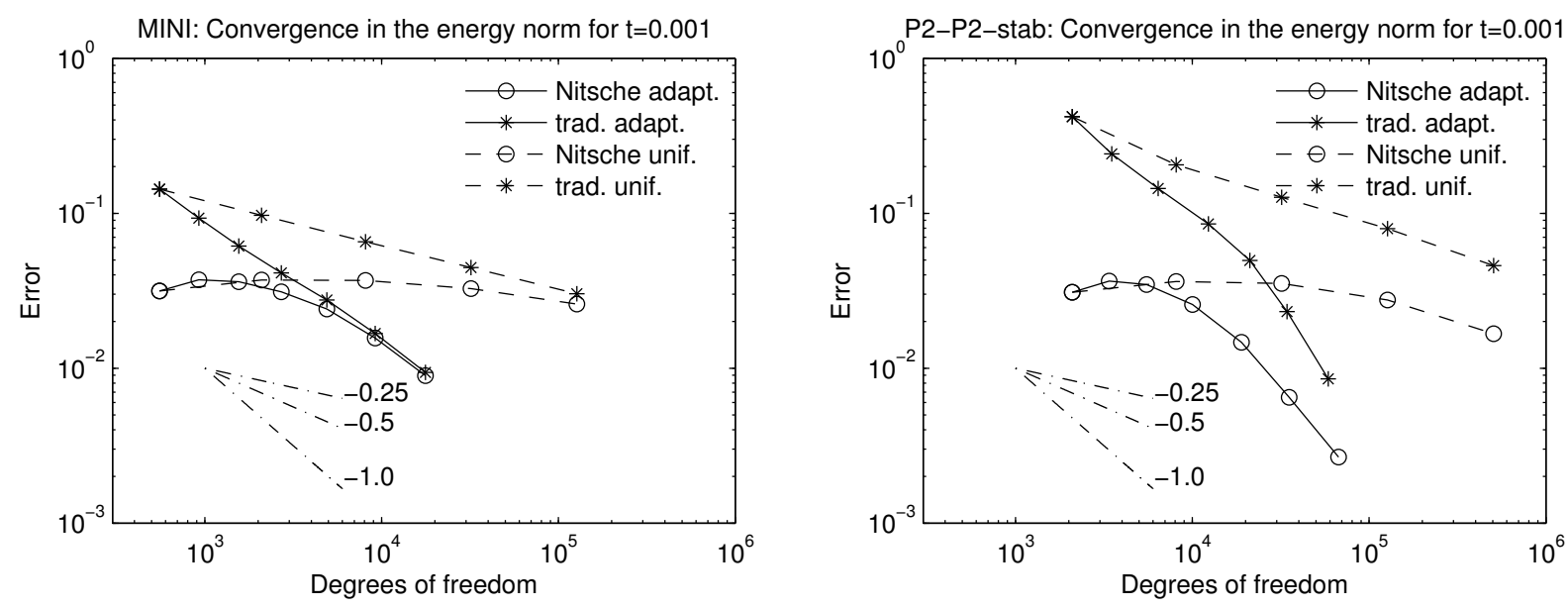

Figure 6: The convergence of the different finite element methods w.r.t. the number of degrees of freedom using adaptive refinement. Both Nitsche's and the traditional boundary conditions are applied. For comparison also the convergence using the uniform refinement is shown. The reference slopes of $-0.25,-0.5$ and -1.0 correspond to $\mathcal{O}(\sqrt{h}), \mathcal{O}(h)$ and $\mathcal{O}\left(h^{2}\right)$ rates of convergence. 

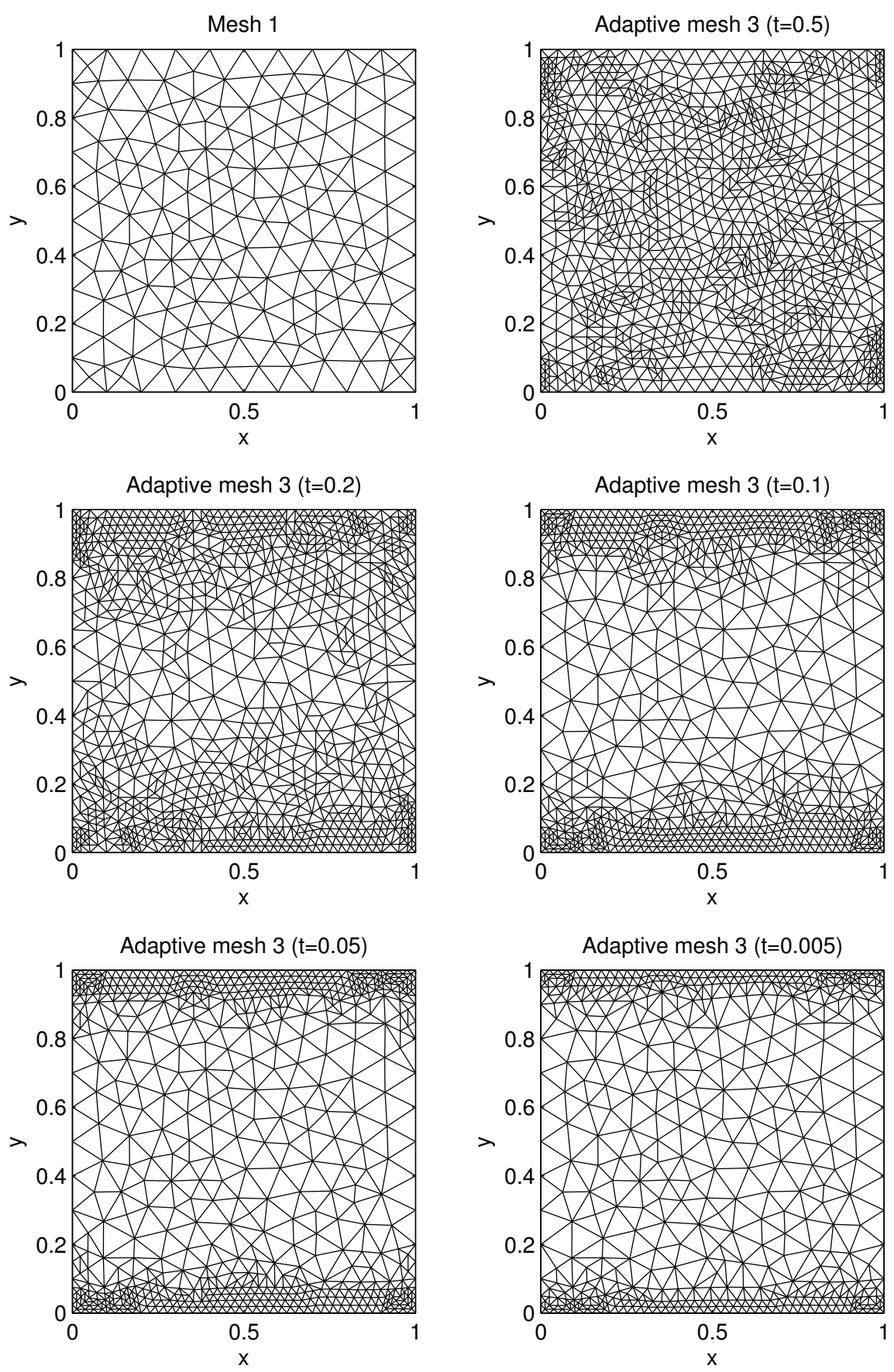

Figure 7: Adaptive meshes with the MINI element using Nitsche's method for various values of parameter $t$. The mesh size in the initial mesh is $h=0.1$. For $t \approx 1$ the solution is smooth hence the adaptive refinement is roughly uniform. For small $t$ the refinement is mostly near the boundaries where the solution has steep changes. 

(continued from the back cover)

A563 Dmitri Kuzmin, Sergey Korotov

Goal-oriented a posteriori error estimates for transport problems

February 2009

A562 Antti H. Niemi

A bilinear shell element based on a refined shallow shell model

December 2008

A561 Antti Hannukainen, Sergey Korotov, Michal Krizek

On nodal superconvergence in 3D by averaging piecewise linear, bilinear, and trilinear $\mathrm{FE}$ approximations

December 2008

A560 Sampsa Pursiainen

Computational methods in electromagnetic biomedical inverse problems

November 2008

A559 Sergey Korotov, Michal Krizek, Jakub Solc

On a discrete maximum principle for linear $F E$ solutions of elliptic problems with a nondiagonal coefficient matrix

November 2008

A558 José Igor Morlanes, Antti Rasila, Tommi Sottinen

Empirical evidence on arbitrage by changing the stock exchange

December 2008

A557 Mika Juntunen, Rolf Stenberg

Analysis of finite element methods for the Brinkman problem

April 2009

A556 Lourenço Beirão da Veiga, Jarkko Niiranen, Rolf Stenberg

A posteriori error analysis for the Morley plate element with general boundary conditions

December 2008

A555 Juho Könnö, Rolf Stenberg

Finite element analysis of composite plates with an application to the paper cockling problem

December 2008 
HELSINKI UNIVERSITY OF TECHNOLOGY INSTITUTE OF MATHEMATICS RESEARCH REPORTS

The reports are available at $h t t p: / / m a t h . t k k . f i / r e p o r t s /$.

The list of reports is continued inside the back cover.

A568 Olavi Nevanlinna

Computing the spectrum and representing the resolvent

April 2009

A567 Antti Hannukainen, Sergey Korotov, Michal Krizek

On a bisection algorithm that produces conforming locally refined simplicial meshes

April 2009

A566 Mika Juntunen, Rolf Stenberg

A residual based a posteriori estimator for the reaction-diffusion problem

February 2009

A565 Ehsan Azmoodeh, Yulia Mishura, Esko Valkeila

On hedging European options in geometric fractional Brownian motion market model

February 2009

A564 Antti H. Niemi

Best bilinear shell element: flat, twisted or curved?

February 2009

ISBN 978-951-22-9860-0 (print)

ISBN 978-951-22-9861-7 (PDF)

ISSN 0784-3143 (print)

ISSN 1797-5867 (PDF) 